\title{
Modified Anesthesia Protocol for Electroconvulsive Therapy Permits Reduction in Aerosol-Generating Bag-Mask Ventilation during the COVID-19 Pandemic
}

\author{
James Luccarelli ${ }^{a, b} \quad$ Claudia Fernandez-Robles ${ }^{c}$ Carlos Fernandez-Robles ${ }^{a}$ \\ Ryan J. Horvath ${ }^{d}$ Sheri Berg ${ }^{d}$ Thomas H. McCoy ${ }^{a}$ Stephen J. Seiner ${ }^{b}$ \\ Michael E. Henrya \\ a Department of Psychiatry, Massachusetts General Hospital, Boston, MA, USA; b Department of Psychiatry, \\ McLean Hospital, Belmont, MA, USA; ' Department of Anesthesiology, Yale School of Medicine, New Haven, CT, USA; \\ ${ }^{\mathrm{d}}$ Department of Anesthesia Critical Care and Pain Medicine, Massachusetts General Hospital, Boston, MA, USA
}

\section{Keywords}

Electroconvulsive therapy · General anesthesia $\cdot$ Mechanical ventilation $\cdot$ COVID-19. Cohort studies

\begin{abstract}
Introduction: Electroconvulsive therapy $(E C T)$ is a critical procedure in psychiatric treatment, but as typically delivered involves the use of bag-mask ventilation (BMV), which during the COVID-19 pandemic exposes patients and treatment staff to potentially infectious aerosols. Objective: To demonstrate the utility of a modified anesthesia protocol for ECT utilizing preoxygenation by facemask and withholding the use of BMV for only those patients who desaturate during the apneic period. Methods: This chart review study analyzes patients who were treated with ECT using both the traditional and modified anesthesia protocols. Results: A total of 106 patients were analyzed, of whom 51 (48.1\%) required BMV using the new protocol. Of clinical factors, only patient BMI was significantly associated with the require-
\end{abstract}

karger@karger.com

(c) 2020 S. Karger AG, Basel

www.karger.com/pps

Karger" ment for BMV. Mean seizure duration reduced from $52.0 \pm$ 22.4 to $46.6 \pm 17.1 \mathrm{~s}$, but seizure duration was adequate in all cases. No acute physical, respiratory, or psychiatric complications occurred during treatment. Conclusions: A modified anesthesia protocol reduces the use of BMV by more than $50 \%$, while retaining adequate seizure duration.

(c) 2020 S. Karger AG, Basel

\section{Introduction}

Electroconvulsive therapy (ECT) is a critical treatment for psychiatric and neurologic illnesses [1], and its use is associated with reduced rates of hospital readmission [2] and all-cause mortality [3] among recipients. To decrease the physical risks of the therapeutic seizure, ECT is performed under general anesthesia and neuromuscular blockade, with bag-mask ventilation (BMV) for respiratory support during the resulting apneic period $[4,5]$. Patients may also be hyperventilated prior to ECT stimula- 
tion to induce hypocarbia and increase seizure duration $[6,7]$, albeit with increased risk of cerebral vasoconstriction [8]. These anesthesia protocols have essentially eliminated the risk of fractures during the procedure [9] and are associated with an overall mortality rate during ECT that is less than that of other procedures performed under general anesthesia [10]. While safe in ordinary clinical circumstances, BMV generates aerosols [11], which have the potential to carry COVID-19 virus in the air and on surfaces for hours [12]. During the COVID-19 pandemic, with some patients asymptomatically carrying the virus [13], the use of BMV exposes staff and patients to infectious risks. As a means of retaining access to this critical psychiatric procedure while improving safety, we developed a modified clinical protocol for ECT treatments, including an anesthesia protocol prioritizing rigorous preoxygenation to reduce the requirement for BMV. This study analyzes the effectiveness of this procedure, and the clinical characteristics of patients who were successfully treated without BMV.

\section{Materials and Methods}

The overarching goal was to retain access to ECT for any patients who required the treatment, including inpatients and those who had not been treated with the procedure before. For inpatients, screening was conducted in accordance with facility protocols, which have changed over the course of the pandemic. All inpatients were clinically assessed on admission for signs or symptoms of COVID-19 infection, and as the pandemic continued to intensify, procedures changed to require COVID-19 PCR testing for all patients being admitted to psychiatry regardless of whether ECT was performed. Inpatient units had isolation rooms for patients known or suspected to be COVID-19 positive, and due to the risks of general anesthesia in these patients with diminished respiratory reserves, ECT was not offered to patients known or suspected to be positive for the virus.

For outpatients, all ECT patients were screened by telephone for COVID-19 exposures and symptoms the day before their scheduled treatment and again on the morning prior to treatment. Patients exhibiting any signs or symptoms concerning for COVID-19 infection, or who had exposure to known positive individuals, were referred for screening at a respiratory clinic or with their primary care doctor, and treatments were held until patients were confirmed negative. As with all patients undergoing general anesthesia, patients receiving outpatient ECT require an escort to accompany them home from the hospital. To minimize exposures from these accompanying individuals, each patient was only permitted one support person in the hospital, and that person was not permitted to remain in the treatment or waiting area until the patient was ready to leave the hospital. As our hospital protocols evolved to require all persons within the facility to wear protective facemasks, this rule was applied to all patients and family members as well as staff.

New Anesthesia Protocol for ECT during the COVID-19 Pandemic

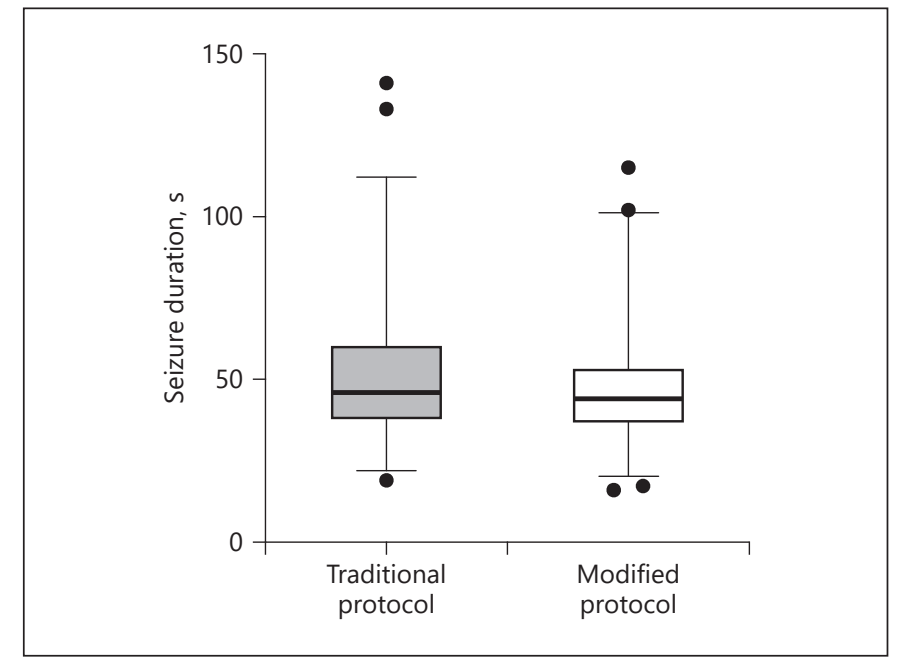

Fig. 1. Plot of seizure duration prior to and following the protocol change reducing the use of bag-mask ventilation. Mean seizure duration reduces from $52.0 \pm 22.4$ to $46.6 \pm 17.1 \mathrm{~s}$ following the protocol change ( $p=0.025$, Wilcoxon signed-rank test). Box and whiskers plots encompass $95 \%$ of the data with remaining values shown as points.

As oxygen therapy by facemask does not generate aerosols [14], before anesthesia induction patients were preoxygenated with a non-rebreather mask for 3-5 min; the mask was left in place through induction, stimulus, and into recovery. Methohexital was used as the induction agent for the majority of patients (typical dose $1 \mathrm{mg} / \mathrm{kg}$, adjusted as needed to maintain adequate anesthesia over the treatment course), with a minority receiving etomidate or ketamine augmentation for inadequate seizures. Once unconsciousness was confirmed by lack of response to stimuli, succinylcholine (initial dose $1 \mathrm{mg} / \mathrm{kg}$, adjusted over the treatment course as needed to maintain adequate paralysis) was then administered for muscle relaxation. There was no modification to anesthesia induction agents or doses. Routine BMV hyperventilation was not provided prior to ECT stimulus. Once muscle relaxation was confirmed by monitoring time and muscle fasciculation, a bite block was inserted, and the non-rebreather left in place. The stimulus was then administered, and the seizure was monitored with two-lead bifrontal or fronto-mastoid EEG. Oxygen saturation was monitored throughout the procedure with pulse oximetry, and BMV was only provided if patients had a significant desaturation, as judged by the treating anesthesiologist. An exact oxygen saturation cut-off was not prescribed given the differences in patient comorbidities and tolerance for possible hypoxia; however, generally an $\mathrm{O}_{2}$ sat of $<85 \%$ triggered BMV sufficient to maintain the oxygen saturation $>85 \%$ and until spontaneous ventilation resumed (generally requiring only 1-3 breaths per patient). When BMV was performed, a HEPA filter (which was saved for use by the same patient on subsequent treatments) was placed between the bag and mask. Ventilation was then performed with careful attention to tight mask seal and with minimum effective tidal volumes and pressures. While induction and ECT stimulus were provided by the full ECT team (anesthesiologist, psychiatrist, and nurse) at one clinical site, if $\mathrm{BMV}$ was provided, all clinical staff except the anesthesiologist ex- 
Table 1. Demographics of the clinical cohort, overall and separated by requirement for bag-mask ventilation using the new protocol

\begin{tabular}{|c|c|c|c|c|}
\hline & Overall & No BMV & BMV required & $p$ value \\
\hline$N$ & 106 & 55 & 51 & \\
\hline Men, $n(\%)$ & $49(46)$ & $22(40)$ & $27(53)$ & 0.24 \\
\hline Women, $n(\%)$ & $57(54)$ & $33(60)$ & $24(47)$ & \\
\hline Age, years & $47.5(18.8)$ & $46.8(19.7)$ & $48.3(18.0)$ & 0.68 \\
\hline BMI & $28.2(6.7)$ & $25.2(4.8)$ & $31.4(7.0)$ & $<0.0001$ \\
\hline \multicolumn{5}{|l|}{ ASA physical status, $n(\%)$} \\
\hline ASA 1 & $14(13)$ & $9(16)$ & $5(10)$ & \\
\hline ASA 2 & $88(83)$ & $42(76)$ & $46(90)$ & \\
\hline ASA 3 & $4(4)$ & $4(7)$ & $0(0)$ & \\
\hline Any pulmonary disease, $n(\%)$ & $25(24)$ & $9(16)$ & $16(31)$ & 0.11 \\
\hline \multicolumn{5}{|l|}{ ECT parameters (new protocol) } \\
\hline Right unilateral, $n(\%)$ & $66(62)$ & $31(56)$ & $35(69)$ & 0.23 \\
\hline Bilateral, $n(\%)$ & $40(38)$ & $24(44)$ & $16(31)$ & \\
\hline Ultrabrief pulse, $n(\%)$ & $38(36)$ & $15(27)$ & $23(45)$ & 0.069 \\
\hline Brief pulse, $n(\%)$ & $68(64)$ & $40(73)$ & $28(55)$ & \\
\hline Charge, $\mathrm{mC}$ & $431.6(157.6)$ & $458.0(159.8)$ & $403.2(151.8)$ & 0.074 \\
\hline \multicolumn{5}{|l|}{ Anesthetic, $n(\%)$} \\
\hline Methohexital & $93(88)$ & $46(84)$ & $47(92)$ & \\
\hline Etomidate & $6(6)$ & $3(5)$ & $3(6)$ & \\
\hline Ketamine and methohexital & $7(7)$ & $6(11)$ & $1(2)$ & \\
\hline
\end{tabular}

Values for age, BMI, and charge are expressed as mean (SD). BMI is the body mass index, defined as the weight in kilograms divided by the square of the height in meters. Pulmonary diseases are any noted on anesthesia exam, and in this sample include obstructive sleep apnea $(n=11)$, asthma $(n=10)$, COPD $(n=3)$, and bronchopulmonary dysplasia $(n=1)$. ECT parameters are for the treatment utilizing the preoxygenation protocol, with ultrabrief pulse defined as a pulse width $<0.5 \mathrm{~ms}$ and brief pulse $0.5-2 \mathrm{~ms}$. All $p$ values are for comparison between patients with no BMV versus those requiring BMV. Two-tailed unpaired $t$ tests were used for continuous variables and Fischer's exact test for dichotomous variables.

ited the room after stimulus and prior to ventilation. At the other clinical site, psychiatry and nursing staff remained in the room during BMV, but all personnel wore N95 respirators and eye protection during all treatments. If a patient required BMV as a result of a desaturation, then it was provided in all subsequent treatments according to the traditional protocol. Under the traditional and modified protocols, all patients received low-dose propofol at the end of the seizure to reduce postictal agitation. This procedure became routine at our institutions in late-March 2020 as the COVID-19 pandemic intensified.

This chart review study analyzes a cohort of patients who received ECT using both the new and traditional anesthesia protocols. The primary study outcomes are the requirement for BMV and the duration of EEG seizure in the last treatment using the old protocol and the first treatment using the new one. Patients were excluded from the study if they were under 18 years of age or if they had not received ECT in the preceding 6 weeks using the traditional ECT anesthesia protocol. No patients were categorially excluded from the new protocol as a result of pulmonary or other medical complications. All treatments utilized a Mecta Spectrum 5000Q (Tualatin, OR, USA). Categorical variables are compared using Fischer's exact test and continuous variables are compared using two-sided $t$ tests or Wilcoxon signed-rank test using Prism (v 8.4.0, San Diego, CA, USA). This study was approved by the Partners Institutional Review Board.

\section{Results}

A total of 106 patients were treated with both the traditional and modified anesthesia procedures; of these, only $51(48.1 \%)$ required BMV using the new protocol. Those who required BMV were similar to those who did not in age, gender, physical comorbidities, and ECT treatment parameters, but those requiring BMV had significantly higher mean BMI (Table 1). Mean seizure duration was shorter using the new protocol, reducing from $52.0 \pm$ 22.4 to $46.6 \pm 17.1 \mathrm{~s}$ following the protocol change $(p=$ 0.025, Wilcoxon signed-rank test; Fig. 1). No acute physical, respiratory, or psychiatric complications occurred during treatment. 


\section{Discussion/Conclusion}

Given the potentially life-saving nature of ECT and the lack of therapeutic alternatives in many cases, ECT is decidedly not an elective procedure $[15,16]$, and patients must be able to continue to access the technique despite the COVID-19 pandemic. Evidence from the 2003 SARS-CoV-1 pandemic indicates a substantial burden of trauma-spectrum and affective disorders among survivors even a year out from treatment [17], and emerging evidence from this pandemic likewise points to high rates of adverse mental health outcomes among healthcare providers treating COVID-19 patients [18]. These individuals will need continued access to the full range of psychiatric treatments, including ECT, and so modifications to clinical protocols that minimize the infectious risk of the procedure are critical. At our study sites, this involves a multidimensional approach, including rigorous screening of all patients for infectious symptoms prior to treatment, minimization of contact from accompanying family members, increased utilization of personal protective equipment, and modification to anesthesia protocols.

Our modified anesthesia procedure using preoxygenation by non-rebreather mask is associated with a $51.9 \%$ reduction in the requirement for BMV in patients undergoing ECT. Those patients who did require BMV were similar clinically to those who did not except for BMI, and this protocol was successfully used with patients 18-91 years old. While patients with baseline respiratory compromise might be expected to be less tolerant of apnea, in our study population the rate of baseline pulmonary disease was not different in the populations that did and did not require BMV, and all patients with ASA 3 physical classification were successfully treated without BMV. Nonetheless, there may be some patients, for example those on baseline oxygen, who may be of such high risk of desaturation that they should be provided ventilation throughout treatment as a matter of course. Our finding of elevated BMI as significantly predictive of desaturation during apnea is consistent with modeling studies in which obesity reduces time to desaturation following succinylcholine administration from $8.7 \mathrm{~min}$ (for a $70-\mathrm{kg}$ adult) to $3.1 \mathrm{~min}$ (for a $127-\mathrm{kg}$ adult) [19].

EEG seizure duration was reduced slightly using the new procedure, but in all cases was above the recommended 15-s minimum duration [20]. This protocol does not permit therapeutic hyperventilation, but the evidence for this technique is weak, and other factors, including changes in ECT stimulus intensity, can be used to modify seizure duration [6]. Moreover, some of the decrease in seizure duration may result from the known anticonvulsant effects of ECT itself $[21,22]$, and not from changes in anesthesia. Separating these effects would be better accomplished using a crossover study design, with some patients receiving the modified anesthesia protocol before being switched to the traditional one. As this crossover design would lead to increased exposure to BMV-generated aerosols, it was deemed too risky during the present COVID-19 pandemic.

Preoxygenation with 100\% oxygen replaces nitrogen in the lungs and tissue and greatly increases total body oxygen stores, which effectively creates a reservoir that can be drawn down during the apneic period from anesthesia induction through paralysis and until resumption of spontaneous ventilation [23]. Preoxygenation is routinely used to prolong time to hypoxemia in procedures such as rapid sequence intubation (RSI), with randomized trials finding non-inferiority for non-rebreather masks relative to bag-valve masks for this purpose [24]. Our results suggest that a similar preoxygenation protocol may be of use during ECT and other short-duration procedures, thereby reducing exposure of clinicians, patients, and the environment to potentially infectious aerosols. Of note, even with BMV throughout the period of apnea, rates of hypoxemia (defined as $\mathrm{SPO}_{2}$ $<90 \%$ ) during ECT are up to $29 \%$, with obesity and increased seizure duration significantly increasing the probability of desaturation [25].

Limitations of the study include an absence of data on psychiatric outcomes and cognitive side effects using the new protocol. As ECT involves multiple treatments administered serially, these outcomes will require more time to become apparent, and longer-duration study is ongoing. As decreased systemic oxygen saturation is correlated with decreased cerebral oxygenation [26], this theoretically creates increased risk of adverse outcomes in those patients who desaturate with this protocol. Additionally, we did not systematically record the number of BMV breaths delivered to those patients requiring them. Our experience was that most patients only required $1-3$ breaths, consistent with a prior study where rates of desaturation were associated with small differences in the number of breaths given during ECT [27], but we are unable to quantify the average number given. Moreover, we did not have a precise desaturation target before administering BMV, but rather allowed for the discretion of the treating anesthesiologist while recommending a target $\mathrm{SPO}_{2}>85 \%$. As numerous patient 
factors affect the rate of desaturation and recovery [19] we felt prescriptive targets would not accurately reflect individualized patient risk, although this complicates extrapolation of this technique to other patient populations.

ECT protocols differ among regions, and optimal treatment delivery likely differs based on factors including local COVID-19 incidence, availability of appropriate PPE, staff experience, and characteristics of the typical patient population. At our centers, our overarching goal was to minimize airway manipulation and aerosol generation. Others might reasonably prioritize avoidance of hypoxemia, and so might prefer methods such as endotracheal intubation, supraglottic airway placement, high flow nasal cannula, or non-invasive ventilation, which provide more reliable oxygen delivery at the cost of additional aerosol generation [28-30]. As present evidence is uncertain about the relative aerosol risk of $\mathrm{BMV}$ versus these interventions, any may be reasonable to use particularly for subsequent treatments of patients who desaturate with the procedure described here. This is consistent with Society for Neuroscience in Anesthesiology \& Critical Care guidelines which recommend minimization of BMV and consideration of alternative oxygenation strategies [31]. Likewise, a RSI procedure in which anesthetic and muscle relaxant are given simultaneously may reduce the duration of apnea and thus the need for BMV. As RSI may come with increased risk of awareness during anesthesia [32], a complication that may be especially problematic for ECT given the requirement for multiple treatments, we elected to use a typical sequential procedure of anesthetic followed by muscle relaxant.

\section{Statement of Ethics}

This chart review study was approved by the Partners Healthcare IRB.

\section{Disclosure Statement}

Dr. McCoy receives research funding from the Stanley Center at the Broad Institute, the Brain and Behavior Research Foundation (26489), Telefonica Alfa, National Institute on Mental Health (Supplement to R01MH104488), and National Institute of Mental Health (R56MH115187). All other authors have no conflicts of interest to declare.

\section{Funding Sources}

This work was supported by the National Institute of Mental Health (R25MH094612; J.L.). The funder had no role in the design and conduct of the study; collection, management, analysis, and interpretation of the data; preparation, review, or approval of the manuscript; and decision to submit the manuscript for publication.

\section{Author Contributions}

Dr. J. Luccarelli had full access to all of the data in the study and takes responsibility for the integrity of the data and the accuracy of the data analysis. Concept and design: Clinical intervention was developed by C. Fernandez-Robles and was adapted for use in other institutions by C. Fernandez-Robles, R.J. Horvath, S. Berg, M.E. Henry, and S.J. Seiner. Acquisition, analysis, or interpretation of data: All authors. Drafting of the manuscript: J. Luccarelli and M.E. Henry. Critical revision of the manuscript for important intellectual content: all authors. Statistical analysis: J. Luccarelli, T.H. McCoy. Administrative, technical, or material support: S.J. Seiner, M.E. Henry. Supervision: M.E. Henry.

\section{References}

1 Sackeim HA. Modern Electroconvulsive Therapy: Vastly Improved yet Greatly Underused. JAMA Psychiatry. 2017 Aug;74(8): 779-80.

2 Slade EP, Jahn DR, Regenold WT, Case BG. Association of Electroconvulsive Therapy With Psychiatric Readmissions in US Hospitals. JAMA Psychiatry. 2017 Aug;74(8):798804.

3 Munk-Olsen T, Laursen TM, Videbech $\mathrm{P}$, Mortensen PB, Rosenberg R. All-cause mortality among recipients of electroconvulsive therapy: register-based cohort study. Br J Psychiatry. 2007 May;190(5):435-9.
4 Mirzakhani H, Guchelaar HJ, Welch CA, Cusin C, Doran ME, MacDonald TO, et al. Minimum Effective Doses of Succinylcholine and Rocuronium During Electroconvulsive Therapy: A Prospective, Randomized, Crossover Trial. Anesth Analg. 2016 Sep;123(3): 587-96.

5 Bryson EO, Aloysi AS, Farber KG, Kellner $\mathrm{CH}$. Individualized Anesthetic Management for Patients Undergoing Electroconvulsive Therapy: A Review of Current Practice. Anesth Analg. 2017 Jun;124(6):1943-56.

6 Gómez-Arnau J, de Arriba-Arnau A, Correas-Lauffer J, Urretavizcaya M. Hyperventilation and electroconvulsive therapy: A literature review. Gen Hosp Psychiatry. 2018 Jan Feb;50:54-62.
7 de Arriba-Arnau A, Dalmau A, Soria V, Salvat-Pujol N, Ribes C, Sánchez-Allueva A, et al. Protocolized hyperventilation enhances electroconvulsive therapy. J Affect Disord. 2017 Aug;217:225-32.

8 Saito S, Kadoi Y, Nihishara F, Aso C, Goto F. End-tidal carbon dioxide monitoring stabilized hemodynamic changes during ECT. J ECT. 2003 Mar;19(1):26-30.

9 Luccarelli J, Henry ME, McCoy TH Jr. Quantification of fracture rate during electroconvulsive therapy (ECT) using state-mandated reporting data. Brain Stimul. 2020 May - Jun; 13(3):523-4 
10 Tørring N, Sanghani SN, Petrides G, Kellner $\mathrm{CH}$, Østergaard SD. The mortality rate of electroconvulsive therapy: a systematic review and pooled analysis. Acta Psychiatr Scand. 2017 May;135(5):388-97.

11 Chan MTV, Chow BK, Lo T, Ko FW, Ng SS, Gin T, et al. Exhaled air dispersion during bag-mask ventilation and sputum suctioning - Implications for infection control. Sci Rep. 2018 Jan;8(1): 198.

12 van Doremalen N, Bushmaker T, Morris DH, Holbrook MG, Gamble A, Williamson BN, et al. Aerosol and Surface Stability of SARS-CoV-2 as Compared with SARSCoV-1. N Engl J Med. 2020 Apr;382(16): 1564-1567.

13 Zhu J, Ji P, Pang J, Zhong Z, Li H, He C, et al Clinical characteristics of 3,062 COVID-19 patients: a meta-analysis. J Med Virol. 2020 Apr. https://doi.org/10.1002/jmv.25884.

14 Simonds AK, Hanak A, Chatwin M, Morrell M, Hall A, Parker KH, et al. Evaluation of droplet dispersion during non-invasive ventilation, oxygen therapy, nebuliser treatment and chest physiotherapy in clinical practice: implications for management of pandemic influenza and other airborne infections. Health Technol Assess. 2010 Oct; 14(46):131172.

15 Espinoza RT, Kellner CH, McCall WV. ECT during COVID-19: An Essential Medical Procedure - Maintaining Service Viability and Accessibility. J ECT. 2020 Jun;36(2):78-79.

16 Sienaert P, Lambrichts S, Popleu L, Van Gerven E, Buggenhout S, Bouckaert F. Electroconvulsive Therapy During COVID-19-Times: Our Patients Cannot Wait. Am J Geriatr Psychiatry. 2020 Apr:S1064-7481(20)30297-9.

17 Lee AM, Wong JG, McAlonan GM, Cheung V, Cheung C, Sham PC, et al. Stress and psy- chological distress among SARS survivors 1 year after the outbreak. Can J Psychiatry. 2007 Apr;52(4):233-40.

18 Lai J, Ma S, Wang Y, Cai Z, Hu J, Wei N, et al. Factors Associated With Mental Health Outcomes Among Health Care Workers Exposed to Coronavirus Disease 2019. JAMA Netw Open. 2020 Mar;3(3):e203976-203976.

19 Benumof JL, Dagg R, Benumof R. Critical hemoglobin desaturation will occur before return to an unparalyzed state following $1 \mathrm{mg} /$ $\mathrm{kg}$ intravenous succinylcholine. Anesthesiology. 1997 Oct;87(4):979-82.

20 Committ AP, Weiner RD. Practice of Electroconvulsive Therapy: Recommendations for Treatment, Training, and Privileging. 2nd ed. Washington (D.C): Amer Psychiatric Pub Inc; 2001.368 pp.

21 Coffey CE, Lucke J, Weiner RD, Krystal AD, Aque M. Seizure threshold in electroconvulsive therapy (ECT) II. The anticonvulsant effect of ECT. Biol Psychiatry. 1995 Jun;37(11): 777-88.

22 Pande AC, Shea J, Shettar S, Grunhaus LJ, Haskett RF. Effect of hyperventilation on seizure length during electroconvulsive therapy. Biol Psychiatry. 1990 Apr;27(7):799-801.

23 Nimmagadda U, Salem MR, Crystal GJ. Preoxygenation: Physiologic Basis, Benefits, and Potential Risks. Anesth Analg. 2017 Feb; 124(2):507-17.

24 Driver BE, Klein LR, Carlson K, Harrington J, Reardon RF, Prekker ME. Preoxygenation With Flush Rate Oxygen: Comparing the Nonrebreather Mask With the Bag-Valve Mask. Ann Emerg Med. 2018 Mar;71(3): 381-6.

25 Surve R, Bansal S, Sriganesh K, Subbakrishna DK, Thirthalli J, Umamaheswara Rao GS. Incidence and risk factors for oxygen desatura- tion during recovery from modified electroconvulsive therapy: A prospective observational study. J Anaesthesiol Clin Pharmacol. 2015 Jan-Mar;31(1):99-103.

26 Sriganesh K, Jadhav T, Venkataramaiah S, Naveen Kumar C, Armugham S, Philip M, et al. Cerebral Oxygen Saturation During Electroconvulsive Therapy: A Secondary Analysis of a Randomized Crossover Trial. J Neurosurg Anesthesiol. 2018 Oct;30(4):314-8.

27 Swindells SR, Simpson KH. Oxygen saturation during electroconvulsive therapy. $\mathrm{Br} J$ Psychiatry. 1987 May;150(5):695-7.

28 Zhu Y, Kang Y, Wei J, Hu J, Wang C, Wang S. Effect of high-flow nasal cannula versus conventional facemask ventilation for patients undergoing modified electroconvulsive therapy: A randomised controlled, noninferiority trial. Eur J Anaesthesiol. 2019 Apr;36(4): 309-10.

29 Nishihara F, Ohkawa M, Hiraoka H, Yuki N, Saito S. Benefits of the laryngeal mask for airway management during electroconvulsive therapy. J ECT. 2003 Dec;19(4):211-6.

30 Weissman DN, de Perio MA, Radonovich LJ. COVID-19 and Risks Posed to Personnel during Endotracheal Intubation. JAMA. 2020 Apr 27. doi: 10.1001/jama.2020.6627. Epub ahead of print.

31 Flexman AM, Abcejo A, Avitisian R, De Sloovere V, Highton D, Juul N, et al. Neuroanesthesia Practice During the COVID-19 Pandemic: Recommendations from Society for Neuroscience in Anesthesiology \& Critical Care (SNACC). J Neurosurg Anesthesiol. 2020 Jul;32(3):202-209.

32 Kimball D, Kincaide RC, Ives C, Henderson $\mathrm{S}$. Rapid Sequence Intubation from the $\mathrm{Pa}$ tient's Perspective. West J Emerg Med. 2011 Nov;12(4):365-7. 